\title{
Decision Support System for Purchase of Used Cars using Electre Method
}

\author{
Novendra Adisaputra Sinaga ${ }^{1}$, Arifin Tua Purba ${ }^{2}$ \\ ${ }^{1,2}$ Politeknik Bisnis Indonesia, Indonesia \\ E-mail: mrnoven@gmail.com, ArifinTuaPrb20@gmail.com
}

\begin{abstract}
This study aims to build a decision support system that can help facilitate the selection of the right used car for potential buyers. To be able to buy a used car that suits the needs and funds owned by consumers, buyers must consider the many criteria and factors of each used car which consists of various brands that exist today. This study uses the ELECTRE (Elimination and Choice Translation Reality) method. The criteria in the comparison used in this decision support system are documents, cylinder volume, year of production and car price. The ELECTRE method is used in conditions where alternatives that do not meet the criteria are eliminated, and suitable alternatives can be generated. Of the 8 samples of used cars studied, the best recommendation was Ayla (A4) with a total aggregate of 4 and an alternative that was not recommended was Agya (A3) with a total aggregate of 0.
\end{abstract}

Keywords: Decision Making System, Used Car, Electre, Criteria, Alternative.

\section{Introduction}

Along with the times that demand to always be fast in all things, it cannot be denied that indirectly transportation has become the necessity of life for everyone. The car is one of the means of transportation needed at this time. When someone has the desire to buy a car, the question is whether to buy a new car or a used car. To buy a used car requires carefulness in choosing a car that is still in good condition and quality because not all used cars have decreased in quality. The criteria used as the basis for choosing a used car include documents, year of production, cylinder volume, and price. The problem that often occurs when someone buys a used car is that buyers often feel disappointed because the car purchased does not meet the criteria needed by the buyer. This is due to the lack of information obtained by potential buyers. To solve this problem, a decision support system was created using the ELECTRE (Elimination and Choice Translation Reality) method where each criterion would be compared so that the buyer would receive recommendations from this system according to the criteria he had chosen. This method can be used to determine whether the car to be purchased is suitable or not with the required qualifications. This is because the ELECTRE method is able to provide the best alternative to the car according to the needs of the buyer.

\section{Literature Review}

\subsection{Decision Support System}

Decision support systems are interactive information systems that provide modeling information and manipulate system data that are used for decision making in semi-structured and unstructured situations where no one is certain about how decisions should be made (Yuliarifin, 2019). DSS is usually built or used for decision making. DSS applications use a CBIS (computer based information system) that is flexible and interactive and can be adapted and developed to support solutions to specific management problems that are not structured. DSS applications using data provide an easy user interface and can incorporate decision-making thinking. DSS is more intended to support management in doing analytical work in less structured situations and with clear criteria. DSS is not intended to automate decision making but provides an interactive tool that enables decision-makers to perform various analyzes using the available models. The goals of a decision support system are: Decision support systems are interactive information systems that provide modeling information and manipulate system data that are used for decision making in semi-structured and unstructured situations where no one is certain about how decisions should be made (Yuliarifin, 2019). DSS is usually built or used for decision making. DSS applications use a CBIS (computer based information system) that is flexible and interactive and can be adapted and developed to support solutions to specific management problems that are not structured. DSS applications using data provide an easy user interface and can incorporate decision-making thinking. DSS is more intended to support management in doing analytical work in less structured situations and with clear criteria. DSS is not intended to automate decision making but provides an interactive tool 
that enables decision-makers to perform various analyzes using the available models. The goals of a decision support

system are :

a. Assisting management in making decisions on semi-structured problems.

b. Provides support for the manager's judgment rather than being intended to replace the manager's function.

c. Increasing the effectiveness of decisions made by managers is more than improving their efficiency.

d. Computing speed, computers enable decision makers to do a lot of computation quickly at low cost.

e. Increased productivity. Building a decision-making group, especially experts, can be very expensive. Computerized support can reduce group size and allow members to be in a variety of different locations (saves on travel costs).

f. Quality support. Computers can improve the quality of what is made.

g. Competitive. Management and company empowerment. Competitive pressures make the decision-making task difficult. Decision-making technology can create significant empowerment by allowing a person to make good decisions quickly, even if they have less knowledge.

Overcome cognitive limitations in processing and storage. The human brain has a limited ability to process and store information. People sometimes find it difficult to remember and use information in an error-free way (Yulianti et.al, 2012).

\section{Method}

According to Janko and Bernoider (2005), Electre is a multi-criteria decision-making method based on the concept of outranking using paired comparisons of alternatives based on each appropriate criterion. The Electre method is used in conditions where alternatives that do not meet the criteria are eliminated, and suitable alternatives can be generated. In other words, Electre is used for cases with many alternatives, but only a few criteria involved. An alternative is said to dominate the other alternatives if one or more of its criteria exceeds (compared to the criteria of the other alternatives) and is the same as the remaining criteria (Kusumadewi et al, 2006). The steps taken in solving the problem using the Electre method are as follows:

1. Determine the Decision Matrix

In the available columns, there is a decision criteria matrix (n) and a row on the alternative form (m) as an initial stage and a basis for processing decision support.

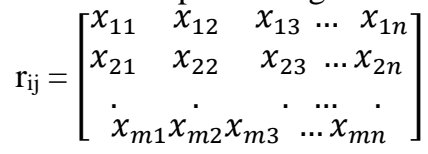

2. Normalization Decision Matrix

The decision matrix will be normalized using a formula and produce a normalized model:

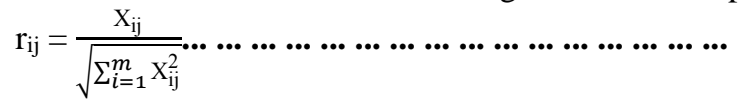

Description :

$$
\begin{array}{llll}
\mathrm{i} & =1,2,3, \ldots, \mathrm{m} & \mathrm{m} & =\text { Number of Alternative } \\
\mathrm{j} & =1,2,3, \ldots, \mathrm{n} & \mathrm{n} & =\text { Number of Criteria }
\end{array}
$$

The results of the decision matrix process are normalized, as shown below :

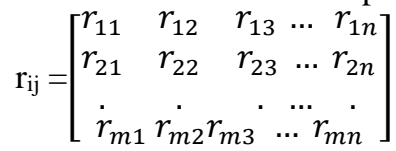

3. Determining Weighting On That Has Normalized Matrix

Each column in the matrix $\mathrm{R}$ multiplied by the weight $(\mathrm{Wj})$ determined by the decision maker can be seen below.

Where $\mathrm{V}$ is :

$$
v_{i j}=W_{i} X_{i j}
$$




$$
\mathrm{v}_{\mathrm{ij}}=\left[\begin{array}{ccccc}
v_{11} & v_{12} & v_{13} & \ldots & v_{1 n} \\
v_{21} & v_{22} & v_{23} & \ldots & v_{2 n} \\
\dot{v}_{m 1} & v_{m 2} & v_{m 3} & \ldots & v_{m n}
\end{array}\right]
$$

4. Determine the set of Concordance and Discordance

The concordance set $\left\{C_{k l}\right\}$ states that the computation of the weight criterion of $A_{k}$ is possibly better than that of $\mathrm{A}_{1}$.

$$
C_{k l}=\left\{j \mid V_{k j} \geq V_{i j}\right\}
$$

Description :

$\mathrm{C}_{\mathrm{kl}} \quad=$ Row concordance value column $\mathrm{k} 1$

$\mathrm{k}, \mathrm{l}=1,2, \ldots, \mathrm{m} \quad \mathrm{k} \neq 1$

$\mathrm{j} \quad=1,2, \ldots, \mathrm{n}$

The set of discordance $\left\{D_{k 1}\right\}$ is written as follows:

Description:

$$
D_{k l}=\left\{j \mid V_{k j} \leq V_{i j}\right\}
$$

$\mathrm{D}_{\mathrm{k} 1}=$ row concordance value column $\mathrm{k} 1$

$\mathrm{k}, 1=1,2, \ldots, \mathrm{m}$

$\mathrm{k} \neq 1$

$\mathrm{j} \quad=1,2, \ldots, \mathrm{n}$

5. Calculate Matrix Concordance and Discordance

To calculate or determine the concordance matrix is to increase the weight contained in the concordance matrix.

$$
C_{k l}=\sum_{j \in C_{k l}} W_{j}
$$

To determine the discordance matrix is to divide the maximum difference between the criteria entered into the discordance set with the highest difference between the values that exist in all criteria.

$$
D_{k l}=\frac{\max \left\{\left|V_{k j}-V_{i j}\right|\right\} j \in C_{k l}}{\max \left\{\left|V_{k j}-V_{i j}\right|\right\} v j}
$$

Matrix D is also the result of $\mathrm{m} \times \mathrm{m}$ and does not take the values of column 1 and row $\mathrm{k}$, array $\mathrm{d}$ as follows:

$$
\mathrm{d}_{\mathrm{ij}}=\left[\begin{array}{cccc}
- & d_{12} & \ldots & d_{1 n} \\
d_{21} & - & \ldots & d_{2 n} \\
d_{m 1} & d_{m 2} & \ldots & -
\end{array}\right]
$$

6. Determining Dominant Concordance Matrix and Dominant Discordance This array can form the upper threshold value (treshold) $\mathrm{C}$. The formula can produce the value $\mathrm{c}$.

$$
\underline{\mathrm{c}}=\frac{\sum_{k=1}^{m} \sum_{l=1}^{m} C_{k l}}{m(m-1)}
$$

The alternative $A_{k}$ can have a chance to dominate $A_{l}$ if the concordance index $C_{k l}$ exceeds the upper threshold value $\mathrm{C}$ with $\mathrm{C}_{\mathrm{kl}} \geq \mathrm{C}$ and the dominant concordance matrix element $\mathrm{F}$ is defined as:

$$
f_{k l}=\frac{1, j i k a C_{k l} \geq C}{0, j i k a C_{k l}<C}
$$

The same is done for the dominant matrix of discordance matrix $\mathrm{G}$ with a threshold value of $\mathrm{D}$. The following formula can give a value of $\mathrm{D}$.

$$
\underline{\mathrm{d}}=\frac{\sum_{k=1}^{m} \sum_{l=1}^{m} d_{k l}}{m(m-1)}
$$

Matriks Element discorden dominan $\mathrm{G}$ defined as :

$$
g_{k l}=\frac{1, j i k a d_{k l} \geq d}{0, j i k a d_{k l}<d}
$$

7. Determine Aggregate DominanceMatrix

Model $\mathrm{E}$ as the dominant total matrix is a matrix where each element is multiplied between the matrix $\mathrm{F}$ and the element equation $\mathrm{G}$.

$$
e_{k l}=f_{k l} * g_{k l}
$$

8. Elimination of Less Favorable Alternatives

47 
In this section, the elimination of alternatives that have a value of 1 at least in the aggregated dominant matrix is carried out. The result of this elimination is the alternative with the highest value of 1 in the aggregate dominance matrix.

\section{Result}

1. Manual calculation methods ELECTRE

In this study, 4 used car samples were used, namely Innova (A1), Terios (A2), Agya (A3), Ayla (A4), Calya (A5), Xenia (A6), Yaris (A7) dan Mobilio (A8) as an alternative to manual calculation with ELECTRE method. Rating matches each alternative on each criterion, rated with one to five, namely :
$1=$ Very Bad
$4=$ Good
$2=\mathrm{Bad}$
5 = Very Good
$3=$ Enough

The following are the criteria that can be chosen by the user

Table 1 Document Criteria Preference Value (C1)

\begin{tabular}{|c|c|}
\hline Nilai & Keterangan \\
\hline 1 & Masa STNK $<=1$ tahun \\
\hline 2 & Masa STNK $>1$ tahun dan $<=2$ tahun \\
\hline 3 & Masa STNK $>2$ tahun dan $<=3$ tahun \\
\hline 4 & Masa STNK $>3$ tahun dan $<=4$ tahun \\
\hline 5 & Masa STNK $>4$ tahun \\
\hline
\end{tabular}

Table 2 Preference Value Criteria for Production Year (C2)

\begin{tabular}{|c|c|}
\hline Nilai & Keterangan \\
\hline 1 & $<=2000$ \\
\hline 2 & $>2000$ dan $<=2005$ \\
\hline 3 & $>2005$ dan $<=2010$ \\
\hline 4 & $>2010$ dan $<=2015$ \\
\hline 5 & $>2015$ \\
\hline
\end{tabular}

Table 3 Value Preference Criteria Cylinder Volume (C3)

\begin{tabular}{|c|c|}
\hline Nilai & Keterangan \\
\hline 1 & $<=1000 \mathrm{cc}$ \\
\hline 2 & $>1000 \mathrm{cc} \mathrm{dan}<=1500 \mathrm{cc}$ \\
\hline 3 & $>1500 \mathrm{ccdan}<=2000 \mathrm{cc}$ \\
\hline 4 & $>2000 \mathrm{cc} \mathrm{dan}<=2500 \mathrm{cc}$ \\
\hline 5 & $>2500 \mathrm{cc}$ \\
\hline
\end{tabular}

Table 4 Value Preference Criteria Price (C4)

\begin{tabular}{|c|c|}
\hline Nilai & Keterangan \\
\hline 1 & $>$ Rp. 500.000 .000 \\
\hline 2 & <=Rp. 500.000.000.000 dan >Rp. 300.000 .000 \\
\hline 3 & <=Rp. 300.000 .000 dan >Rp. 150.000 .000 \\
\hline 4 & <=Rp. 150.000 .000 dan >Rp. 100.000 .000 \\
\hline 5 & <=Rp. 100.000 .000 \\
\hline
\end{tabular}

The criterion importance level (preference weight) is also assessed by one to five, namely:

$1=$ Very Low

$4=$ Hight

$2=$ Low

$5=$ Very Hight

$3=$ Enough

In this study, decision makers give preference weights as follows:
a. Document Criteria $(\mathrm{C} 1)=5$
c. Cylinder Volume Criteria $(\mathrm{C} 3)=3$
b. Production Year Criteria $(\mathrm{C} 2)=4$
d. Price Criteria $(\mathrm{C} 4)=4$

With the result that $\mathrm{W}=5,4,3,4$

The next steps are as follows:

1. Determine the Decision Matrix 
Table 5 Decision Matrix

\begin{tabular}{|c|c|c|c|c|}
\hline \multirow{2}{*}{ Alternative } & \multicolumn{4}{|c|}{ Criteria } \\
\cline { 2 - 5 } & C1 & C2 & C3 & C4 \\
\hline $\mathbf{A 1}$ & 1 & 3 & 3 & 4 \\
\hline $\mathbf{A 2}$ & 2 & 4 & 2 & 4 \\
\hline $\mathbf{A 3}$ & 4 & 5 & 2 & 4 \\
\hline $\mathbf{A 4}$ & 3 & 5 & 2 & 5 \\
\hline $\mathbf{A 5}$ & 2 & 5 & 2 & 4 \\
\hline $\mathbf{A 6}$ & 2 & 5 & 2 & 4 \\
\hline $\mathbf{A 7}$ & 1 & 4 & 2 & 4 \\
\hline $\mathbf{A 8}$ & 1 & 5 & 2 & 4 \\
\hline
\end{tabular}

2. Normalization of the Decision Matrix

$$
\begin{aligned}
& \mathrm{R}_{11}=\frac{1}{1^{2}+2^{2}+4^{2}+3^{2}+2^{2}+2^{2}+1^{2}+1^{2}}=\frac{1}{\sqrt{40}}=\frac{1}{6,3246}=0,1581 \\
& \mathrm{R}_{21}=\frac{2}{1^{2}+2^{2}+4^{2}+3^{2}+2^{2}+2^{2}+1^{2}+1^{2}}=\frac{2}{\sqrt{40}}=\frac{2}{6,3246}=0,3162 \\
& \mathrm{R}_{31}=\frac{4}{1^{2}+2^{2}+4^{2}+3^{2}+2^{2}+2^{2}+1^{2}+1^{2}}=\frac{4}{\sqrt{40}}=\frac{4}{6,3246}=0,6325 \\
& \mathrm{R}_{41}=\frac{2}{1^{2}+2^{2}+4^{2}+3^{2}+2^{2}+2^{2}+1^{2}+1^{2}}=\frac{3}{\sqrt{40}}=\frac{3}{6,3246}=0,4743 \\
& \mathrm{R}_{51}=\frac{2}{1^{2}+2^{2}+4^{2}+3^{2}+2^{2}+2^{2}+1^{2}+1^{2}}=\frac{2}{\sqrt{40}}=\frac{2}{6,3246}=0,3162 \\
& \mathrm{R}_{61}=\frac{1}{1^{2}+2^{2}+4^{2}+3^{2}+2^{2}+2^{2}+1^{2}+1^{2}}=\frac{1}{\sqrt{40}}=\frac{2}{6,3246}=0,3162 \\
& \mathrm{R}_{71}=\frac{1}{1^{2}+2^{2}+4^{2}+3^{2}+2^{2}+2^{2}+1^{2}+1^{2}}=\frac{1}{\sqrt{40}}=\frac{1}{6,3246}=0,1581 \\
& \mathrm{R}_{81}=\frac{4}{1^{2}+2^{2}+4^{2}+3^{2}+2^{2}+2^{2}+1^{2}+1^{2}}=\frac{4}{\sqrt{40}}=\frac{1}{6,3246}=0,1581 \\
& \mathrm{R}_{12}=\frac{3}{3^{2}+4^{2}+5^{2}+5^{2}+5^{2}+5^{2}+4^{2}+5^{2}}=\frac{4}{\sqrt{166}}=\frac{3}{12,8841}=0,2328 \\
& \mathrm{R}_{22}=\frac{4}{3^{2}+4^{2}+5^{2}+5^{2}+5^{2}+5^{2}+4^{2}+5^{2}}=\frac{4}{\sqrt{166}}=\frac{4}{12,8841}=0,3105 \\
& \mathrm{R}_{32}=\frac{5}{3^{2}+4^{2}+5^{2}+5^{2}+5^{2}+5^{2}+4^{2}+5^{2}}=\frac{5}{\sqrt{166}}=\frac{5}{12,8841}=0,3881 \\
& \mathrm{R}_{42}=\frac{5}{3^{2}+4^{2}+5^{2}+5^{2}+5^{2}+5^{2}+4^{2}+5^{2}}=\frac{5}{\sqrt{166}}=\frac{5}{12,8841}=0,3881 \\
& \mathrm{R}_{52}=\frac{5}{3^{2}+4^{2}+5^{2}+5^{2}+5^{2}+5^{2}+4^{2}+5^{2}}=\frac{5}{\sqrt{166}}=\frac{5}{12,8841}=0,3881 \\
& \mathrm{R}_{62}=\frac{5}{3^{2}+4^{2}+5^{2}+5^{2}+5^{2}+5^{2}+4^{2}+5^{2}}=\frac{5}{\sqrt{166}}=\frac{5}{12,8841}=0,3881 \\
& \mathrm{R}_{72}=\frac{4}{3^{2}+4^{2}+5^{2}+5^{2}+5^{2}+5^{2}+4^{2}+5^{2}}=\frac{4}{\sqrt{166}}=\frac{4}{12,8841}=0,3105 \\
& \mathrm{R}_{82}=\frac{5}{3^{2}+4^{2}+5^{2}+5^{2}+5^{2}+5^{2}+4^{2}+5^{2}}=\frac{5}{\sqrt{166}}=\frac{3}{12,8841}=0,3881
\end{aligned}
$$

So that the normalized $\mathrm{R}$ matrix is obtained:

$$
X=\left[\begin{array}{llll}
1 & 3 & 3 & 4 \\
2 & 4 & 2 & 4 \\
4 & 5 & 2 & 4 \\
3 & 5 & 2 & 5 \\
2 & 5 & 2 & 4 \\
2 & 5 & 2 & 4 \\
1 & 4 & 2 & 4 \\
1 & 5 & 2 & 4
\end{array}\right]
$$

$$
\begin{aligned}
& \mathrm{R}_{13}=\frac{3}{3^{2}+2^{2}+2^{2}+1^{2}+2^{2}+2^{2}+2^{2}+2^{2}}=\frac{3}{\sqrt{34}}=\frac{3}{5,8301}=0,5145 \\
& \mathrm{R}_{23}=\frac{2}{3^{2}+2^{2}+2^{2}+1^{2}+2^{2}+2^{2}+2^{2}+2^{2}}=\frac{2}{\sqrt{34}}=\frac{2}{5,8301}=0,3430 \\
& \mathrm{R}_{33}=\frac{2}{3^{2}+2^{2}+2^{2}+1^{2}+2^{2}+2^{2}+2^{2}+2^{2}}=\frac{2}{\sqrt{34}}=\frac{2}{5,8301}=0,3430 \\
& \mathrm{R}_{43}=\frac{1}{3^{2}+2^{2}+2^{2}+1^{2}+2^{2}+2^{2}+2^{2}+2^{2}}=\frac{1}{\sqrt{34}}=\frac{1}{5,8301}=0,1715 \\
& \mathrm{R}_{53}=\frac{2}{3^{2}+2^{2}+2^{2}+1^{2}+2^{2}+2^{2}+2^{2}+2^{2}}=\frac{2}{\sqrt{34}}=\frac{2}{5,8301}=0,3430 \\
& \mathrm{R}_{63}=\frac{2}{3^{2}+2^{2}+2^{2}+1^{2}+2^{2}+2^{2}+2^{2}+2^{2}}=\frac{2}{\sqrt{34}}=\frac{2}{5,8301}=0,3430 \\
& \mathrm{R}_{73}=\frac{2}{3^{2}+2^{2}+2^{2}+1^{2}+2^{2}+2^{2}+2^{2}+2^{2}}=\frac{2}{\sqrt{34}}=\frac{2}{5,8301}=0,3430 \\
& \mathrm{R}_{83}=\frac{2}{3^{2}+2^{2}+2^{2}+1^{2}+2^{2}+2^{2}+2^{2}+2^{2}}=\frac{2}{\sqrt{34}}=\frac{2}{5,8301}=0,3430 \\
& \mathrm{R}_{14}=\frac{4}{4^{2}+4^{2}+4^{2}+5^{2}+4^{2}+4^{2}+4^{2}+4^{2}}=\frac{4}{\sqrt{137}}=\frac{4}{11,7046}=0,3417 \\
& \mathrm{R}_{24}=\frac{4}{4^{2}+4^{2}+4^{2}+5^{2}+4^{2}+4^{2}+4^{2}+4^{2}}=\frac{4}{\sqrt{137}}=\frac{4}{11,7046}=0,3417 \\
& \mathrm{R}_{34}=\frac{4}{4^{2}+4^{2}+4^{2}+5^{2}+4^{2}+4^{2}+4^{2}+4^{2}}=\frac{4}{\sqrt{137}}=\frac{4}{11,7046}=0,3417 \\
& \mathrm{R}_{44}=\frac{5}{4^{2}+4^{2}+4^{2}+5^{2}+4^{2}+4^{2}+4^{2}+4^{2}}=\frac{5}{\sqrt{137}}=\frac{5}{11,7046}=0,4272 \\
& \mathrm{R}_{54}=\frac{4}{4^{2}+4^{2}+4^{2}+5^{2}+4^{2}+4^{2}+4^{2}+4^{2}}=\frac{4}{\sqrt{137}}=\frac{4}{11,7046}=0,3417 \\
& \mathrm{R}_{64}=\frac{4}{4^{2}+4^{2}+4^{2}+5^{2}+4^{2}+4^{2}+4^{2}+4^{2}}=\frac{4}{\sqrt{137}}=\frac{4}{11,7046}=0,3417 \\
& \mathrm{R}_{74}=\frac{4}{4^{2}+4^{2}+4^{2}+5^{2}+4^{2}+4^{2}+4^{2}+4^{2}}=\frac{4}{\sqrt{137}}=\frac{4}{11,7046}=0,3417 \\
& \mathrm{R}_{84}=\frac{4}{4^{2}+4^{2}+4^{2}+5^{2}+4^{2}+4^{2}+4^{2}+4^{2}}=\frac{4}{\sqrt{137}}=\frac{4}{11,7046}=0,3417
\end{aligned}
$$

Table 6 Normalization Results

\begin{tabular}{|c|c|c|c|c|}
\hline \multirow{2}{*}{ Alternative } & \multicolumn{4}{|c|}{ Criteria } \\
\cline { 2 - 5 } & $\mathbf{C 1}$ & $\mathbf{C 2}$ & $\mathbf{C 3}$ & $\mathbf{C 4}$ \\
\hline $\mathbf{A 1}$ & 0,1581 & 0,2328 & 0,5145 & 0,3417 \\
\hline $\mathbf{A 2}$ & 0,3162 & 0,3105 & 0,3430 & 0,3417 \\
\hline $\mathbf{A 3}$ & 0,6325 & 0,3881 & 0,3430 & 0,3417 \\
\hline $\mathbf{A 4}$ & 0,4743 & 0,3881 & 0,1715 & 0,4272 \\
\hline $\mathbf{A 5}$ & 0,3162 & 0,3881 & 0,3430 & 0,3417 \\
\hline $\mathbf{A 6}$ & 0,3162 & 0,3881 & 0,3430 & 0,3417 \\
\hline $\mathbf{A 7}$ & 0,1581 & 0,3105 & 0,3430 & 0,3417 \\
\hline $\mathbf{A 8}$ & 0,1581 & 0,3881 & 0,3430 & 0,3417 \\
\hline
\end{tabular}

3. Determine the weighting of the Normalized Matrix 


$$
\mathrm{R}=\left[\begin{array}{lllll}
0,1581 & 0,2328 & 0,5145 & 0,3417 \\
0,3162 & 0,3105 & 0,3430 & 0,3417 \\
0,6325 & 0,3881 & 0,3430 & 0,3417 \\
0,4743 & 0,3881 & 0,1715 & 0,4272 \\
0,3162 & 0,3881 & 0,3430 & 0,3417 \\
0,3162 & 0,3881 & 0,3430 & 0,3417 \\
0,1581 & 0,3105 & 0,3430 & 0,3417 \\
0,1581 & 0,3881 & 0,3430 & 0,3417
\end{array}\right] \times \quad \mathrm{W} \quad=\quad \mathrm{V}=\left[\begin{array}{lllll}
\text { So that the matrix V is obtained: } \\
1,5811 & 1,2418 & 1,0290 & 1,3670 \\
3,1623 & 1,5523 & 1,0290 & 1,3670 \\
2,3717 & 1,5523 & 0,5145 & 1,7087 \\
1,5811 & 1,5523 & 1,0290 & 1,3670 \\
1,5811 & 1,5523 & 1,0290 & 1,3670 \\
0,7906 & 1,2418 & 1,0290 & 1,3670 \\
2,7906 & 1,5523 & 1,0290 & 1,3670
\end{array}\right]
$$

4. Determine the set Concordance and Discordance
a. Concordance
$\mathrm{C}_{51}=\left\{\mathrm{j}, \mathrm{V}_{5 \mathrm{j}} \geq \mathrm{V}_{1 \mathrm{j}}\right\}=\{1,2,4\}$
$\mathrm{C}_{12}=\left\{\mathrm{j}, \mathrm{V}_{1 \mathrm{j}} \geq \mathrm{V}_{2 \mathrm{j}}\right\}=\{3,4\}$
$\mathrm{C}_{13}=\left\{\mathrm{j}, \mathrm{V}_{1 \mathrm{j}} \geq \mathrm{V}_{3 \mathrm{j}}\right\}=\{3,4\}$
$\mathrm{C}_{52}=\left\{\mathrm{j}, \mathrm{V}_{5 \mathrm{j}} \geq \mathrm{V}_{2 \mathrm{j}}\right\}=\{1,2,3,4\}$
$\mathrm{C}_{14}=\left\{\mathrm{j}, \mathrm{V}_{1 \mathrm{j}} \geq \mathrm{V}_{4 \mathrm{j}}\right\}=\{3,4\}$
$\mathrm{C}_{53}=\left\{\mathrm{j}, \mathrm{V}_{5 \mathrm{j}} \geq \mathrm{V}_{3 \mathrm{j}}\right\}=\{2,3,4\}$
$\mathrm{C}_{15}=\left\{\mathrm{j}, \mathrm{V}_{1 \mathrm{j}} \geq \mathrm{V}_{5 \mathrm{j}}\right\}=\{3,4\}$
$\mathrm{C}_{54}=\left\{\mathrm{j}, \mathrm{V}_{5 \mathrm{j}} \geq \mathrm{V}_{4 \mathrm{j}}\right\}=\{2,3\}$
$\mathrm{C}_{16}=\left\{\mathrm{j}, \mathrm{V}_{1 \mathrm{j}} \geq \mathrm{V}_{6 \mathrm{j}}\right\}=\{3,4\}$
$\mathrm{C}_{56}=\left\{\mathrm{j}, \mathrm{V}_{5 \mathrm{j}} \geq \mathrm{V}_{6 \mathrm{j}}\right\}=\{1,2,3,4\}$
$\mathrm{C}_{17}=\left\{\mathrm{j}, \mathrm{V}_{1 \mathrm{j}} \geq \mathrm{V}_{7 \mathrm{j}}\right\}=\{1,3,4\}$
$\mathrm{C}_{57}=\left\{\mathrm{j}, \mathrm{V}_{5 \mathrm{j}} \geq \mathrm{V}_{7 \mathrm{j}}\right\}=\{1,2,3,4\}$
$\mathrm{C}_{18}=\left\{\mathrm{j}, \mathrm{V}_{1 \mathrm{j}} \geq \mathrm{V}_{8 \mathrm{j}}\right\}=\{1,3,4\}$
$\mathrm{C}_{58}=\left\{\mathrm{j}, \mathrm{V}_{5 \mathrm{j}} \geq \mathrm{V}_{8 \mathrm{j}}\right\}=\{1,2,3,4\}$
$\mathrm{C}_{21}=\left\{\mathrm{j}, \mathrm{V}_{2 \mathrm{j}} \geq \mathrm{V}_{1 \mathrm{j}}\right\}=\{1,2,4\}$
$\mathrm{C}_{61}=\left\{\mathrm{j}, \mathrm{V}_{6 \mathrm{j}} \geq \mathrm{V}_{1 \mathrm{j}}\right\}=\{1,2,4\}$
$\mathrm{C}_{23}=\left\{\mathrm{j}, \mathrm{V}_{2 \mathrm{j}} \geq \mathrm{V}_{3 \mathrm{j}}\right\}=\{3,4\}$
$\mathrm{C}_{62}=\left\{\mathrm{j}, \mathrm{V}_{6 \mathrm{j}} \geq \mathrm{V}_{2 \mathrm{j}}\right\}=\{1,2,3,4\}$
$\mathrm{C}_{24}=\left\{\mathrm{j}, \mathrm{V}_{2 \mathrm{j}} \geq \mathrm{V}_{4 \mathrm{j}}\right\}=\{3,4\}$
$\mathrm{C}_{63}=\left\{\mathrm{j}, \mathrm{V}_{6 \mathrm{j}} \geq \mathrm{V}_{3 \mathrm{j}}\right\}=\{2,3,4\}$
$\mathrm{C}_{25}=\left\{\mathrm{j}, \mathrm{V}_{2 \mathrm{j}} \geq \mathrm{V}_{5 \mathrm{j}}\right\}=\{1,3,4\}$
$\mathrm{C}_{64}=\left\{\mathrm{j}, \mathrm{V}_{6 \mathrm{j}} \geq \mathrm{V}_{4 \mathrm{j}}\right\}=\{2,3\}$
$\mathrm{C}_{65}=\left\{\mathrm{j}, \mathrm{V}_{6 \mathrm{j}} \geq \mathrm{V}_{5 \mathrm{j}}\right\}=\{1,2,3,4\}$
$\mathrm{C}_{26}=\left\{\mathrm{j}, \mathrm{V}_{2 \mathrm{j}} \geq \mathrm{V}_{6 \mathrm{j}}\right\}=\{1,3,4\}$
$\mathrm{C}_{67}=\left\{\mathrm{j}, \mathrm{V}_{6 \mathrm{j}} \geq \mathrm{V}_{7 \mathrm{j}}\right\}=\{1,2,3,4\}$
$\mathrm{C}_{27}=\left\{\mathrm{j}, \mathrm{V}_{2 \mathrm{j}} \geq \mathrm{V}_{\mathrm{V}_{\mathrm{j}}}\right\}=\{1,2,3,4\}$
$\mathrm{C}_{68}=\left\{\mathrm{j}, \mathrm{V}_{6 \mathrm{j}} \geq \mathrm{V}_{8 \mathrm{j}}\right\}=\{1,2,3,4\}$
$\mathrm{C}_{28}=\left\{\mathrm{j}, \mathrm{V}_{2 \mathrm{j}} \geq \mathrm{V}_{8 \mathrm{j}}\right\}=\{1,3,4\}$
$\mathrm{C}_{71}=\left\{\mathrm{j}, \mathrm{V}_{7 \mathrm{j}} \geq \mathrm{V}_{1 \mathrm{j}}\right\}=\{1,2,4\}$
$\mathrm{C}_{31}=\left\{\mathrm{j}, \mathrm{V}_{3 \mathrm{j}} \geq \mathrm{V}_{1 \mathrm{j}}\right\}=\{1,2,4\}$
$\mathrm{C}_{72}=\left\{\mathrm{j}, \mathrm{V}_{7 \mathrm{j}} \geq \mathrm{V}_{2 \mathrm{j}}\right\}=\{2,3,4\}$
$\mathrm{C}_{32}=\left\{\mathrm{j}, \mathrm{V}_{3 \mathrm{j}} \geq \mathrm{V}_{2 \mathrm{j}}\right\}=\{1,2,3,4\}$
$\mathrm{C}_{73}=\left\{\mathrm{j}, \mathrm{V}_{7 \mathrm{j}} \geq \mathrm{V}_{3 \mathrm{j}}\right\}=\{3,4\}$
$\mathrm{C}_{34}=\left\{\mathrm{j}, \mathrm{V}_{3 \mathrm{j}} \geq \mathrm{V}_{4 \mathrm{j}}\right\}=\{1,2,3\}$
$\mathrm{C}_{35}=\left\{\mathrm{j}, \mathrm{V}_{3 \mathrm{j}} \geq \mathrm{V}_{5 \mathrm{j}}\right\}=\{1,2,3,4\}$
$\mathrm{C}_{74}=\left\{\mathrm{j}, \mathrm{V}_{7 \mathrm{j}} \geq \mathrm{V}_{4 \mathrm{j}}\right\}=\{3\}$
$\mathrm{C}_{75}=\left\{\mathrm{j}, \mathrm{V}_{7 \mathrm{j}} \geq \mathrm{V}_{5 \mathrm{j}}\right\}=\{3,4\}$
$C_{36}=\left\{j, V_{3 j} \geq V_{6 j}\right\}=\{1,2,3,4\}$
$\mathrm{C}_{76}=\left\{\mathrm{j}, \mathrm{V}_{7 \mathrm{j}} \geq \mathrm{V}_{6 \mathrm{j}}\right\}=\{3,4\}$
$\mathrm{C}_{37}=\left\{\mathrm{j}, \mathrm{V}_{3 \mathrm{j}} \geq \mathrm{V}_{7 \mathrm{j}}\right\}=\{1,2,3,4\}$
$\mathrm{C}_{78}=\left\{\mathrm{j}, \mathrm{V}_{7 \mathrm{j}} \geq \mathrm{V}_{8 \mathrm{j}}\right\}=\{1,3,4\}$
$\mathrm{C}_{38}=\left\{\mathrm{j}, \mathrm{V}_{3 \mathrm{j}} \geq \mathrm{V}_{8 \mathrm{j}}\right\}=\{1,2,3,4\}$
$\mathrm{C}_{81}=\left\{\mathrm{j}, \mathrm{V}_{8 \mathrm{j}} \geq \mathrm{V}_{1 \mathrm{j}}\right\}=\{1,2,4\}$
$\mathrm{C}_{41}=\left\{\mathrm{j}, \mathrm{V}_{4 \mathrm{j}} \geq \mathrm{V}_{1 \mathrm{j}}\right\}=\{1,2,4\}$
$\mathrm{C}_{42}=\left\{\mathrm{j}, \mathrm{V}_{4 \mathrm{j}} \geq \mathrm{V}_{2 \mathrm{j}}\right\}=\{1,2,4\}$
$\mathrm{C}_{82}=\left\{\mathrm{j}, \mathrm{V}_{8 \mathrm{j}} \geq \mathrm{V}_{2 \mathrm{j}}\right\}=\{2,3,4\}$
$\mathrm{C}_{83}=\left\{\mathrm{j}, \mathrm{V}_{8 \mathrm{j}} \geq \mathrm{V}_{3 \mathrm{j}}\right\}=\{2,3,4\}$
$\mathrm{C}_{43}=\left\{\mathrm{j}, \mathrm{V}_{4 \mathrm{j}} \geq \mathrm{V}_{3 \mathrm{j}}\right\}=\{2,4\}$
$\mathrm{C}_{45}=\left\{\mathrm{j}, \mathrm{V}_{4 \mathrm{j}} \geq \mathrm{V}_{5 \mathrm{j}}\right\}=\{1,2,4\}$
$\mathrm{C}_{46}=\left\{\mathrm{j}, \mathrm{V}_{4 \mathrm{j}} \geq \mathrm{V}_{6 \mathrm{j}}\right\}=\{1,2,4\}$
$\mathrm{C}_{47}=\left\{\mathrm{j}, \mathrm{V}_{4 \mathrm{j}} \geq \mathrm{V}_{7 \mathrm{j}}\right\}=\{1,2,4\}$
$\mathrm{C}_{48}=\left\{\mathrm{j}, \mathrm{V}_{4 \mathrm{j}} \geq \mathrm{V}_{8 \mathrm{j}}\right\}=\{1,2,4\}$
$\mathrm{C}_{84}=\left\{\mathrm{j}, \mathrm{V}_{8 \mathrm{j}} \geq \mathrm{V}_{4 \mathrm{j}}\right\}=\{2,3,4\}$
$\mathrm{C}_{85}=\left\{\mathrm{j}, \mathrm{V}_{8 \mathrm{j}} \geq \mathrm{V}_{5 \mathrm{j}}\right\}=\{2,3,4\}$
$\mathrm{C}_{86}=\left\{\mathrm{j}, \mathrm{V}_{8 \mathrm{j}} \geq \mathrm{V}_{6 \mathrm{j}}\right\}=\{2,3,4\}$
$\mathrm{C}_{87}=\left\{\mathrm{j}, \mathrm{V}_{8 \mathrm{j}} \geq \mathrm{V}_{7 \mathrm{j}}\right\}=\{1,2,3,4\}$

In Order to get the set concordance :

Table 7 Concordance Association

\begin{tabular}{|c|c|c|c|c|c|c|c|c|}
\hline Alternative & \multicolumn{7}{|c|}{ Concordance } \\
\hline $\mathbf{A 1}$ & - & 3,4 & 3,4 & 3,4 & 3,4 & 3,4 & $1,3,4$ & $1,3,4$ \\
\hline $\mathbf{A 2}$ & $1,2,4$ & - & 3,4 & 3,4 & $1,3,4$ & $1,3,4$ & $\begin{array}{c}1,2,3, \\
4\end{array}$ & $1,3,4$ \\
\hline $\mathbf{A 3}$ & $1,2,4$ & $\begin{array}{c}1,2,3 \\
4\end{array}$ & - & $1,2,3$ & $\begin{array}{c}1,2,3, \\
4\end{array}$ & $\begin{array}{c}1,2,3, \\
4\end{array}$ & $\begin{array}{c}1,2,3, \\
4\end{array}$ & $\begin{array}{c}1,2,3, \\
4\end{array}$ \\
\hline $\mathbf{A 4}$ & $1,2,4$ & $1,2,4$ & 2,4 & - & $1,2,4$ & $1,2,4$ & $1,2,4$ & $1,2,4$ \\
\hline $\mathbf{A 5}$ & $1,2,4$ & $\begin{array}{c}1,2,3 \\
4\end{array}$ & $2,3,4$ & 2,3, & - & $\begin{array}{c}1,2,3, \\
4\end{array}$ & $\begin{array}{c}1,2,3 \\
4\end{array}$ & $\begin{array}{c}1,2,3, \\
4\end{array}$ \\
\hline
\end{tabular}


Journal of Computer Networks, Architecture and High Performance Computing e-ISSN 2655-9102, Volume 1, No. 2, Juli 2019, pp 45 -59

\begin{tabular}{|c|c|c|c|c|c|c|c|c|}
\hline $\mathbf{A 6}$ & $1,2,4$ & $\begin{array}{c}1,2,3, \\
4\end{array}$ & $2,3,4$ & 2,3 & $\begin{array}{c}1,2,3, \\
4\end{array}$ & - & $\begin{array}{c}1,2,3, \\
4\end{array}$ & $\begin{array}{c}1,2,3, \\
4\end{array}$ \\
\hline $\mathbf{A 7}$ & $1,2,4$ & $2,3,4$ & 3,4 & 3 & 3,4 & 3,4 & - & $1,3,4$ \\
\hline $\mathbf{A 8}$ & $1,2,4$ & $2,3,4$ & $2,3,4$ & $2,3,4$ & $2,3,4$ & $2,3,4$ & $\begin{array}{c}1,2,3, \\
4\end{array}$ & - \\
\hline
\end{tabular}

b. Discordance

\begin{tabular}{|c|c|}
\hline$D_{12}=\left\{j, V_{1 j}<V_{2 j}\right\}=\{1,2\}$ & $D_{51}=\left\{j, V_{5 j}<V_{1 j}\right\}=\{3\}$ \\
\hline $\mathrm{D}_{13}=\left\{\mathrm{j}, \mathrm{V}_{1 \mathrm{j}}<\mathrm{V}_{3 \mathrm{j}}\right\}=\{1,2\}$ & $D_{52}=\left\{j, V_{5 j}<V_{2 j}\right\}=\{\}$ \\
\hline$D_{14}=\left\{j, V_{1 j}<V_{4 j}\right\}=\{1,2,4\}$ & $\mathrm{D}_{53}=\left\{\mathrm{j}, \mathrm{V}_{5 \mathrm{j}}<\mathrm{V}_{3 \mathrm{j}}\right\}=\{1\}$ \\
\hline$D_{15}=\left\{j, V_{1 j}<V_{5 j}\right\}=\{1,2\}$ & $\mathrm{D}_{54}=\left\{\mathrm{j}, \mathrm{V}_{5 \mathrm{j}}<\mathrm{V}_{4 \mathrm{j}}\right\}=\{1,4\}$ \\
\hline$D_{16}=\left\{j, V_{1 j}<V_{6 j}\right\}=\{1,2\}$ & $\mathrm{D}_{56}=\left\{\mathrm{j}, \mathrm{V}_{5 \mathrm{j}}<\mathrm{V}_{6 \mathrm{j}}\right\}=\{\}$ \\
\hline$D_{17}=\left\{j, V_{1 j}<V_{7 j}\right\}=\{2\}$ & $D_{57}=\left\{j, V_{5 j}<V_{7 j}\right\}=\{\}$ \\
\hline$D_{18}=\left\{j, V_{1 j}<V_{8 j}\right\}=\{2\}$ & $\mathrm{D}_{58}=\left\{\mathrm{j}, \mathrm{V}_{5 \mathrm{j}}<\mathrm{V}_{8 \mathrm{j}}\right\}=\{\}$ \\
\hline$D_{21}=\left\{j, V_{2 j}<V_{1 j}\right\}=\{3\}$ & $D_{61}=\left\{j, V_{6 j}<V_{1 j}\right\}=\{3\}$ \\
\hline$D_{23}=\left\{j, V_{2 j}<V_{3 j}\right\}=\{1,2\}$ & $\mathrm{D}_{62}=\left\{\mathrm{j}, \mathrm{V}_{6 \mathrm{j}}<\mathrm{V}_{2 \mathrm{j}}\right\}=\{\}$ \\
\hline $\mathrm{D}_{24}=\left\{\mathrm{j}, \mathrm{V}_{2 \mathrm{j}}<\mathrm{V}_{4 \mathrm{j}}\right\}=\{1,2,4\}$ & $D_{63}=\left\{j, V_{6 j}<V_{3 j}\right\}=\{1\}$ \\
\hline $\mathrm{D}_{25}=\left\{\mathrm{j}, \mathrm{V}_{2 \mathrm{j}}<\mathrm{V}_{5 \mathrm{j}}\right\}=\{2\}$ & $\mathrm{D}_{64}=\left\{\mathrm{j}, \mathrm{V}_{6 \mathrm{j}}<\mathrm{V}_{4 \mathrm{j}}\right\}=\{1,4\}$ \\
\hline$D_{26}=\left\{j, V_{2 j}<V_{6 j}\right\}=\{2\}$ & $D_{65}=\left\{j, V_{6 j}<V_{5 j}\right\}=\{\}$ \\
\hline $\mathrm{D}_{27}=\left\{\mathrm{j}, \mathrm{V}_{2 \mathrm{j}}<\mathrm{V}_{7 \mathrm{j}}\right\}=\{\}$ & $D_{67}=\left\{j, V_{6 j}<V_{7 j}\right\}=\{\}$ \\
\hline $\mathrm{D}_{28}=\left\{\mathrm{j}, \mathrm{V}_{2 \mathrm{j}}<\mathrm{V}_{1 \mathrm{j}}\right\}=\{2\}$ & $\mathrm{D}_{68}=\left\{\mathrm{j}, \mathrm{V}_{6 \mathrm{j}}<\mathrm{V}_{8 \mathrm{j}}\right\}=\{\}$ \\
\hline$D_{31}=\left\{j, V_{3 j}<V_{1 j}\right\}=\{3\}$ & $\mathrm{D}_{71}=\left\{\mathrm{j}, \mathrm{V}_{7 \mathrm{j}}<\mathrm{V}_{1 \mathrm{j}}\right\}=\{3\}$ \\
\hline$D_{32}=\left\{j, V_{3 j}<V_{2 j}\right\}=\{\}$ & $\mathrm{D}_{72}=\left\{\mathrm{j}, \mathrm{V}_{7 \mathrm{j}}<\mathrm{V}_{2 \mathrm{j}}\right\}=\{1\}$ \\
\hline$D_{34}=\left\{j, V_{3 j}<V_{4 j}\right\}=\{4\}$ & $D_{73}=\left\{j, V_{7 j}<V_{3 j}\right\}=\{1,2\}$ \\
\hline $\mathrm{D}_{35}=\left\{\mathrm{j}, \mathrm{V}_{3 \mathrm{j}}<\mathrm{V}_{5 \mathrm{j}}\right\}=\{\}$ & $\mathrm{D}_{74}=\left\{\mathrm{j}, \mathrm{V}_{7 \mathrm{j}}<\mathrm{V}_{4 \mathrm{j}}\right\}=\{1,2,4$ \\
\hline $\mathrm{D}_{36}=\left\{\mathrm{j}, \mathrm{V}_{3 \mathrm{j}}<\mathrm{V}_{6 \mathrm{j}}\right\}=\{\}$ & $D_{75}=\left\{j, V_{7 j}<V_{5 j}\right\}=\{1,2\}$ \\
\hline $\mathrm{D}_{37}=\left\{\mathrm{j}, \mathrm{V}_{3 \mathrm{j}}<\mathrm{V}_{7 \mathrm{j}}\right\}=\{\}$ & $D_{76}=\left\{j, V_{7 j}<V_{6 j}\right\}=\{1,2\}$ \\
\hline $\mathrm{D}_{38}=\left\{\mathrm{j}, \mathrm{V}_{3 \mathrm{j}}<\mathrm{V}_{8 \mathrm{j}}\right\}=\{\}$ & $D_{78}=\left\{j, V_{7 j}<V_{8 j}\right\}=\{2\}$ \\
\hline $\mathrm{D}_{41}=\left\{\mathrm{j}, \mathrm{V}_{4 \mathrm{j}}<\mathrm{V}_{1 \mathrm{j}}\right\}=\{3\}$ & $\mathrm{D}_{81}=\left\{\mathrm{j}, \mathrm{V}_{8 \mathrm{j}}<\mathrm{V}_{1 \mathrm{j}}\right\}=\{3\}$ \\
\hline $\mathrm{D}_{42}=\left\{\mathrm{j}, \mathrm{V}_{4 \mathrm{j}}<\mathrm{V}_{2 \mathrm{j}}\right\}=\{3\}$ & $\mathrm{D}_{82}=\left\{\mathrm{j}, \mathrm{V}_{8 \mathrm{j}}<\mathrm{V}_{2 \mathrm{j}}\right\}=\{1\}$ \\
\hline$D_{43}=\left\{j, V_{4 j}<V_{3 j}\right\}=\{1,3\}$ & $\mathrm{D}_{83}=\left\{\mathrm{j}, \mathrm{V}_{8 \mathrm{j}}<\mathrm{V}_{3 \mathrm{j}}\right\}=\{1\}$ \\
\hline $\mathrm{D}_{45}=\left\{\mathrm{j}, \mathrm{V}_{4 \mathrm{j}}<\mathrm{V}_{5 \mathrm{j}}\right\}=\{3\}$ & $\mathrm{D}_{84}=\left\{\mathrm{j}, \mathrm{V}_{8 \mathrm{j}}<\mathrm{V}_{4 \mathrm{j}}\right\}=\{1,4\}$ \\
\hline$D_{46}=\left\{j, V_{4 j}<V_{6 j}\right\}=\{3\}$ & $\mathrm{D}_{85}=\left\{\mathrm{j}, \mathrm{V}_{8 \mathrm{j}}<\mathrm{V}_{5 \mathrm{j}}\right\}=\{1\}$ \\
\hline $\mathrm{D}_{47}=\left\{\mathrm{j}, \mathrm{V}_{4 \mathrm{j}}<\mathrm{V}_{7 \mathrm{j}}\right\}=\{3\}$ & $\mathrm{D}_{86}=\left\{\mathrm{j}, \mathrm{V}_{8 \mathrm{j}}<\mathrm{V}_{6 \mathrm{j}}\right\}=\{1\}$ \\
\hline $\mathrm{D}_{48}=\left\{\mathrm{j}, \mathrm{V}_{4 \mathrm{j}}<\mathrm{V}_{8 \mathrm{j}}\right\}=\{3\}$ & $D_{87}=\left\{j, V_{8 j}<V_{7 j}\right\}=\{\}$ \\
\hline
\end{tabular}

In order to obtain the set of discordance:

Tabel 8 Set Discordance

\begin{tabular}{|c|l|l|l|l|l|l|l|l|}
\hline Alternative & \multicolumn{7}{|c|}{ Discordance } \\
\hline $\mathbf{A 1}$ & - & 1,2 & 1,2 & $1,2,4$ & 1,2 & 1,2 & 2 & 2 \\
\hline $\mathbf{A 2}$ & 3 & - & 1,2 & $1,2,4$ & 2 & 2 & 0 & 2 \\
\hline $\mathbf{A 3}$ & 3 & 0 & - & 4 & 0 & 0 & 0 & 0 \\
\hline $\mathbf{A 4}$ & 3 & 3 & 1,3 & - & 3 & 3 & 3 & 3 \\
\hline $\mathbf{A 5}$ & 3 & 0 & 1 & 1,4 & - & 0 & 0 & 0 \\
\hline $\mathbf{A 6}$ & 3 & 0 & 1 & 1,4 & 0 & - & 0 & 0 \\
\hline $\mathbf{A 7}$ & 3 & 1 & 1,2 & $1,2,4$ & 1,2 & 1,2 & - & 2 \\
\hline $\mathbf{A 8}$ & 3 & 1 & 1 & 1,4 & 1 & 1 & 0 & - \\
\hline
\end{tabular}

5. Calculate the Matriks Concordance and Discordance
a. Concordance
$\mathrm{C}_{12}=\mathrm{W}_{3}+\mathrm{W}_{4}$
$=3+4=7$
$\mathrm{C}_{51}=\mathrm{W}_{1}+\mathrm{W}_{2}+\mathrm{W}_{4}$
$=5+4+4$
$=13$

51 
$\mathrm{C}_{13}=\mathrm{W}_{3}+\mathrm{W}_{4}$

$\mathrm{C}_{14}=\mathrm{W}_{3}+\mathrm{W}_{4}$

$\mathrm{C}_{15}=\mathrm{W}_{3}+\mathrm{W}_{4}$

$\mathrm{C}_{16}=\mathrm{W}_{3}+\mathrm{W}_{4}$

$\mathrm{C}_{17}=\mathrm{W}_{1}+\mathrm{W}_{3}+\mathrm{W}_{4}$

$\mathrm{C}_{18}=\mathrm{W}_{1}+\mathrm{W}_{3}+\mathrm{W}_{4}$

$\mathrm{C}_{21}=\mathrm{W}_{1}+\mathrm{W}_{2}+\mathrm{W}_{4}$

$\mathrm{C}_{23}=\mathrm{W}_{3}+\mathrm{W}_{4}$

$\mathrm{C}_{24}=\mathrm{W}_{3}+\mathrm{W}_{4}$

$\mathrm{C}_{25}=\mathrm{W}_{1}+\mathrm{W}_{3}+\mathrm{W}_{4}$

$\mathrm{C}_{26}=\mathrm{W}_{1}+\mathrm{W}_{3}+\mathrm{W}_{4}$

$\mathrm{C}_{27}=\mathrm{W}_{1}+\mathrm{W}_{2}+\mathrm{W}_{3}+\mathrm{W}_{4}$

$\mathrm{C}_{28}=\mathrm{W}_{1}+\mathrm{W}_{3}+\mathrm{W}_{4}=5+3+4$

$\mathrm{C}_{31}=\mathrm{W}_{1}+\mathrm{W}_{2}+\mathrm{W}_{4} \quad=5+4+4$

$\mathrm{C}_{32}=\mathrm{W}_{1}+\mathrm{W}_{2}+\mathrm{W}_{3}+\mathrm{W}_{4}=5+4+3+4$

$\mathrm{C}_{34}=\mathrm{W}_{1}+\mathrm{W}_{2}+\mathrm{W}_{3}+\mathrm{W}_{4}=5+4+3+4$

$\mathrm{C}_{35}=\mathrm{W}_{1}+\mathrm{W}_{2}+\mathrm{W}_{3}=5+4+3$

$\mathrm{C}_{36}=\mathrm{W}_{1}+\mathrm{W}_{2}+\mathrm{W}_{3}+\mathrm{W}_{4}=5+4+3+4$

$\mathrm{C}_{37}=\mathrm{W}_{1}+\mathrm{W}_{2}+\mathrm{W}_{3}+\mathrm{W}_{4}=5+4+3+4$

$\mathrm{C}_{38}=\mathrm{W}_{1}+\mathrm{W}_{2}+\mathrm{W}_{3}+\mathrm{W}_{4}=5+4+3+4$

$\mathrm{C}_{41}=\mathrm{W}_{1}+\mathrm{W}_{2}+\mathrm{W}_{4}=5+4+4$

$\mathrm{C}_{42}=\mathrm{W}_{1}+\mathrm{W}_{2}+\mathrm{W}_{4}=5+4+4$

$\mathrm{C}_{43}=\mathrm{W}_{2}+\mathrm{W}_{4}$

$\mathrm{C}_{45}=\mathrm{W}_{1}+\mathrm{W}_{2}+\mathrm{W}_{4}$

$\mathrm{C}_{46}=\mathrm{W}_{1}+\mathrm{W}_{2}+\mathrm{W}_{4}$

$\mathrm{C}_{47}=\mathrm{W}_{1}+\mathrm{W}_{2}+\mathrm{W}_{4}$

$\mathrm{C}_{48}=\mathrm{W}_{1}+\mathrm{W}_{2}+\mathrm{W}_{4}$
$=4+4$

$=5+4+4$

$=5+4+4$

$=5+4+4$

$=5+4+4$
$\mathrm{C}_{52}=\mathrm{W}_{1}+\mathrm{W}_{2}+\mathrm{W}_{3}+\mathrm{W}_{4}=5+4+3+4=16$

$\mathrm{C}_{53}=\mathrm{W}_{2}+\mathrm{W}_{3}+\mathrm{W}_{4}=4+3+4=11$

$\mathrm{C}_{54}=\mathrm{W}_{2}+\mathrm{W}_{3}=4+3=7$

$\mathrm{C}_{56}=\mathrm{W}_{1}+\mathrm{W}_{2}+\mathrm{W}_{3}+\mathrm{W}_{4}=5+4+3+4=16$

$\mathrm{C}_{57}=\mathrm{W}_{1}+\mathrm{W}_{2}+\mathrm{W}_{3}+\mathrm{W}_{4}=5+4+3+4=16$

$\mathrm{C}_{58}=\mathrm{W}_{1}+\mathrm{W}_{2}+\mathrm{W}_{3}+\mathrm{W}_{4}=5+4+3+4=16$

$\mathrm{C}_{61}=\mathrm{W}_{1}+\mathrm{W}_{2}+\mathrm{W}_{4}=5+4+4=13$

$\mathrm{C}_{62}=\mathrm{W}_{1}+\mathrm{W}_{2}+\mathrm{W}_{3}+\mathrm{W}_{4}=5+4+3+4=16$

$\mathrm{C}_{63}=\mathrm{W}_{2}+\mathrm{W}_{3}+\mathrm{W}_{4}=4+3+4=11$

$\mathrm{C}_{64}=\mathrm{W}_{2}+\mathrm{W}_{3}=4+3=7$

$\mathrm{C}_{65}=\mathrm{W}_{1}+\mathrm{W}_{2}+\mathrm{W}_{3}+\mathrm{W}_{4}=5+4+3+4=16$

$\mathrm{C}_{67}=\mathrm{W}_{1}+\mathrm{W}_{2}+\mathrm{W}_{3}+\mathrm{W}_{4}=5+4+3+4=16$

$\mathrm{C}_{68}=\mathrm{W}_{1}+\mathrm{W}_{2}+\mathrm{W}_{3}+\mathrm{W}_{4}=5+4+3+4=16$

$\mathrm{C}_{71}=\mathrm{W}_{1}+\mathrm{W}_{2}+\mathrm{W}_{4}=5+4+4=13$

$\mathrm{C}_{72}=\mathrm{W}_{2}+\mathrm{W}_{3}+\mathrm{W}_{4} \quad=4+3+4=11$

$\mathrm{C}_{73}=\mathrm{W}_{3}+\mathrm{W}_{4}=3+4=7$

$\mathrm{C}_{74}=\mathrm{W}_{3}$

$\mathrm{C}_{75}=\mathrm{W}_{3}+\mathrm{W}_{4}$

$\mathrm{C}_{76}=\mathrm{W}_{3}+\mathrm{W}_{4}$

$\mathrm{C}_{78}=\mathrm{W}_{1}+\mathrm{W}_{3}+\mathrm{W}_{4}$

$\mathrm{C}_{81}=\mathrm{W}_{1}+\mathrm{W}_{2}+\mathrm{W}_{4}$

$\mathrm{C}_{82}=\mathrm{W}_{2}+\mathrm{W}_{3}+\mathrm{W}_{4}$

$\mathrm{C}_{83}=\mathrm{W}_{2}+\mathrm{W}_{3}+\mathrm{W}_{4}$

$\mathrm{C}_{84}=\mathrm{W}_{2}+\mathrm{W}_{3}+\mathrm{W}_{4}$

$\mathrm{C}_{85}=\mathrm{W}_{2}+\mathrm{W}_{3}+\mathrm{W}_{4}$

$\mathrm{C}_{86}=\mathrm{W}_{2}+\mathrm{W}_{3}+\mathrm{W}_{4}$

$=3$

$=3+4=7$

$=3+4=7$

$=5+3+4=12$

$=5+4+4=13$

$=4+3+4=11$

$=4+3+4=11$

$=4+3+4=11$

$=4+3+4=11$

$=13 \quad \mathrm{C}_{87}=\mathrm{W}_{1}+\mathrm{W}_{2}+\mathrm{W}_{3}+\mathrm{W}_{4}=5+4+3+4=16$

So its concordance matrix are:

$\mathrm{C}=\left[\begin{array}{cccccccc}- & 7 & 7 & 3 & 7 & 7 & 12 & 12 \\ 13 & - & 7 & 3 & 12 & 12 & 16 & 12 \\ 13 & 16 & - & 12 & 16 & 16 & 16 & 16 \\ 13 & 13 & 8 & - & 13 & 13 & 13 & 13 \\ 13 & 16 & 11 & 7 & - & 16 & 16 & 16 \\ 13 & 16 & 11 & 7 & 16 & - & 16 & 16 \\ 13 & 11 & 7 & 3 & 7 & 7 & - & 12 \\ 13 & 11 & 11 & 7 & 11 & 11 & 16 & -\end{array}\right]$

\section{b. Discordance}

$\mathrm{d}_{12}=$

$\max \{|0,7906-1,5811| ;|0,9314-1,2418|\}$
$\max \{|0,7906-1,5811| ;|0,9314-1,2418| ;|1,5435-1,0290| ;|1,3670-1,3670|\}$ $\max \{0,7906 ; 0,3105\}$
$=\frac{\max \{0,7906 ; 0,3105 ; 0,5145 ; 0\}}{\operatorname{mox}}$
$=\frac{0,7906}{0,7906}=1$
$\mathrm{d}_{13}=$
$\max \{|0,7906-3,1623| ;|0,9314-1,5523|\}$
$\max \{|0,7906-3,1623| ;|0,9314-1,5523| ;|1,5435-1,0290| ;|1,3670-1,3670|\}$ $=\frac{\max \{2,3717 ; 0,6209\}}{\max \{2,3717 ; 0,6209 ; 0 ; 0\}}$ $=\frac{2,3717}{2,3717}=1$

$\mathrm{d}_{14}=$

$\max \{|0,7906-2,3717| ;|0,9314-1,5523| ;|1,3670-1,7087|\}$

$\max \{|0,7906-2,3717| ;|0,9314-1,5523| ;|1,5435-0,5145| ;|1,3670-1,7087|\}$

$\mathrm{D}_{51}=$

$\max \{|1,0290-1,5435|\}$

$\overline{\max \{|1,5811-0,7906| ;|1,5523-0,9314| ;|1,0290-1,5435| ;|1,3670-1,3670|\}}$ $\max \{0,7906\}$

$=\overline{\max \{0,7906 ; 0,6209 ; 0,5145 ; 0\}}$

$=\frac{0,7906}{0,7906}=1$

$\mathrm{D}_{52}=$

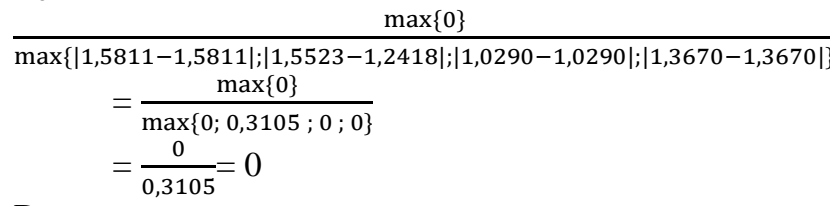

$\mathrm{D}_{53}=$

$\max \{0\}$

$\max \{|1,5811-3,1623| ;|1,5523-1,5523| ;|1,0290-1,0290| ;|1,3670-1,3670|\}$ 


$$
\begin{aligned}
& =\frac{\max \{1,5811 ; 0,6209 ; 0,3147\}}{\max \{1,5811 ; 0,6209 ; 1,0290 ; 0,3417\}} \\
& =\frac{1,5811}{1,5811}=1 \\
& \mathrm{~d}_{15}= \\
& \max \{|0,7906-1,5811| ;|0,9314-1,5523|\} \\
& \max \{|0,7906-1,5811| ;|0,9314-1,5523| ;|1,5435-1,0290| ;|1,3670-1,3670|\} \\
& =\frac{\max \{0,7906 ; 0,6209\}}{\max \{0,7906 ; 0,620 ; 0,5145 ;} \\
& =\overline{\max \{0,7906 ; 0,6209 ; 0,5145 ; 0\}} \\
& =\frac{0,7906}{0,7906}=1 \\
& \mathrm{~d}_{16}= \\
& \max \{|0,7906-1,5811| ;|0,9314-1,5523|\} \\
& \overline{\max \{|0,7906-1,5811| ;|0,9314-1,5523| ;|1,5435-1,0290| ;|1,3670-1,3670|\}} \\
& \max \{0,7906 ; 0,6209\} \\
& =\overline{\max \{0,7906 ; 0,6209 ; 0,5145 ; 0\}} \\
& =\frac{0,7906}{0,7906}=1 \\
& \mathrm{~d}_{17}= \\
& \max \{|0,9314-1,2418|\} \\
& \overline{\max \{|0,7906-0,7906| ;|0,9314-1,2418| ;|1,5435-1,0290| ;|1,3670-1,3670|\}} \\
& =\frac{\max \{0,3105\}}{\max \{0 ; 0,3105 ; 0,5145 ; 0\}} \\
& =\frac{0,3105}{0,5145}=0,6034
\end{aligned}
$$

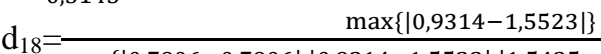

$$
\begin{aligned}
& \begin{array}{l}
\mathrm{d}_{18}=\frac{\max \{|0,7906-0,7906| ;|0,9314-1,5523| ;|1,5435-1,0290| ;|1,3670-1,3670|\}}{\max \{0,6209\}} \\
=
\end{array} \\
& =\frac{\max \{0 ; 0,6209 ; 0,5145 ; 0\}}{\max } \\
& =\frac{0,6209}{0,6209}=1 \\
& \mathrm{~d}_{21}= \\
& \max \{|1,0290-1,5435|\} \\
& \max \{|1,5811-0,7906| ;|1,2418-0,9314| ;|1,0290-1,5435| ;|1,3670-1,3670|\} \\
& =\frac{\max \{0,5145\}}{\max \{0,7906 ; 0,3105 ; 0,51} \\
& =\overline{\max \{0,7906 ; 0,3105 ; 0,5145 ; 0\}} \\
& =\frac{0,5145}{0,7906}=0,6508 \\
& \mathrm{~d}_{23}= \\
& \max \{|1,5811-3,1623| ;|1,2418-1,5523|\} \\
& \max \{|1,5811-3,1623| ;|1,2418-1,5523| ;|1,0290-1,0290| ;|1,3670-1,3670|\} \\
& =\frac{\max \{1,5811 ; 0,3105\}}{\max \{1,5811 ; 0,3105 ; 0 ; 0\}} \\
& =\frac{\max \{1,5811 ; 0,3105 ; 0 ; 0\}}{\operatorname{mox}} \\
& =\frac{1,5811}{1,5811}=1 \\
& \mathrm{~d}_{24}= \\
& \max \{|1,5811-2,3717| ;|1,2418-1,5523| ;|1,3670-1,7087|\} \\
& \overline{\max \{|1,5811-2,3717| ;|1,2418-1,5523| ;|1,0290-0,5145| ;|1,3670-1,7087|\}} \\
& =\frac{\max \{0,7906 ; 0,3105 ; 0,3417\}}{\max \{0,7906 ; 0,3105 ; 0 ; 0,3417\}} \\
& =\frac{\max \{0,7906 ; 0,3105 ; 0 ; 0,3417\}}{\operatorname{mon}} \\
& =\frac{0,7906}{0,7906}=1 \\
& \mathrm{~d}_{25}= \\
& \max \{|1,2418-1,5523|\} \\
& \max \{|1,5811-1,5811| ;|1,2418-1,5523| ;|1,0290-1,0290| ;|1,3670-1,3670|\} \\
& =\frac{\max \{|0,3150|\}}{\max \{0 ; 0,3150 ; 0 ; 0\}} \\
& =\frac{0,3150}{0,3150}=1 \\
& \mathrm{~d}_{26}= \\
& \max \{|1,2418-1,5523|\} \\
& \max \{|1,5811-1,5811| ;|1,2418-1,5523| ;|1,0290-1,0290| ;|1,3670-1,3670|\} \\
& =\frac{\max \{|0,3150|\}}{\max \{0 ; 0,3150 ; 0 ; 0\}} \\
& =\overline{\max \{0 ; 0,3150 ; 0 ; 0\}} \\
& =\frac{0,3150}{0,3150}=1 \\
& =\frac{\max \{0\}}{\max \{0 ; 1,5811 ; 0 ; 0\}} \\
& =\frac{0}{1,5811}=0 \\
& \mathrm{D}_{54}= \\
& \max \{|1,5811-2,3717||1,3670-1,7087|\} \\
& \max \{|1,5811-2,3717| ;|1,5523-1,5523| ;|1,0290-0,5145| ;|1,3670-1,7087|\} \\
& =\frac{\max \{0,7906 ; 0,3417\}}{\max \{0,7906 ; 0 ; 0,5145 ; 0,341} \\
& =\overline{\max \{0,7906 ; 0 ; 0,5145 ; 0,3417\}} \\
& =\frac{0,7906}{0,7906}=1 \\
& \overline{\max \{|1,5811-1,5811| ;|1,5523-1,5523| ;|1,0290-1,0290| ;|1,3670-1,3670|\}} \\
& \max \{0\} \\
& =\overline{\max \{0 ; 0 ; 0 ; 0\}} \\
& =\frac{0}{0}=0 \\
& \mathrm{D}_{57}= \\
& \max \{|1,5811-0,7906| ;|1,5523-1,2418| ;|1,0290-1,0290| ;|1,3670-1,3670|\} \\
& \begin{array}{l}
=\frac{\max \{0\}}{\max \{0,7906 ; 0,3105 ; 0 ; 0\}} \\
=\frac{0}{0,7906}=0
\end{array} \\
& \max \{0\} \\
& =\frac{\max \{0\}}{\max \{0,7906 ; 0 ; 0 ; 0\}} \\
& =\frac{0}{0,7906}=0 \\
& \max \{|1,5811-0,7906| ;|1,5523-0,9314| ;|1,0290-1,5435| ;|1,3670-1,3670|\} \\
& \max \{0,7906\} \\
& =\overline{\max \{0,7906 ; 0,6209 ; 0,5145 ; 0\}} \\
& =\frac{0,7906}{0,7906}=1 \\
& \mathrm{D}_{62}= \\
& \max \{0\} \\
& \overline{\max \{|1,5811-1,5811| ;|1,5523-1,2418| ;|1,0290-1,0290| ;|1,3670-1,3670|\}} \\
& =\frac{\max \{0\}}{\max \{0 ; 0,3105 ; 0 ; 0\}} \\
& =\frac{0}{0,3105}=0 \\
& \mathrm{D}_{63}= \\
& \max \{|1,5811-3,1623| ;|1,5523-1,5523| ;|1,0290-1,0290| ;|1,3670-1,3670|\} \\
& =\frac{\max \{0\}}{\max \{0 ; 1,5811 ; 0 ; 0\}} \\
& =\frac{0}{1,5811}=0 \\
& \mathrm{D}_{64}= \\
& \max \{|1,5811-2,3717||1,3670-1,7087|\} \\
& \max \{|1,5811-2,3717| ;|1,5523-1,5523| ;|1,0290-0,5145| ;|1,3670-1,7087|\} \\
& =\frac{\max \{0,7906 ; 0,3417\}}{\max \{0,7906 ; 0 ; 0,5145 ; 0,341} \\
& =\overline{\max \{0,7906 ; 0 ; 0,5145 ; 0,3417\}} \\
& =\frac{0,7906}{0,7906}=1 \\
& \mathrm{D}_{65}= \\
& \max \{0\} \\
& \max \{|1,5811-1,5811| ;|1,5523-1,5523| ;|1,0290-1,0290| ;|1,3670-1,3670|\} \\
& =\frac{\max \{0\}}{\max \{0 ; 0 ; 0 ; 0\}}
\end{aligned}
$$

53

Journal of Computer Networks, Architecture and High Performance Computing is licensed under a

CreativeCommons Attribution-NoDerivatives 4.0 International License 


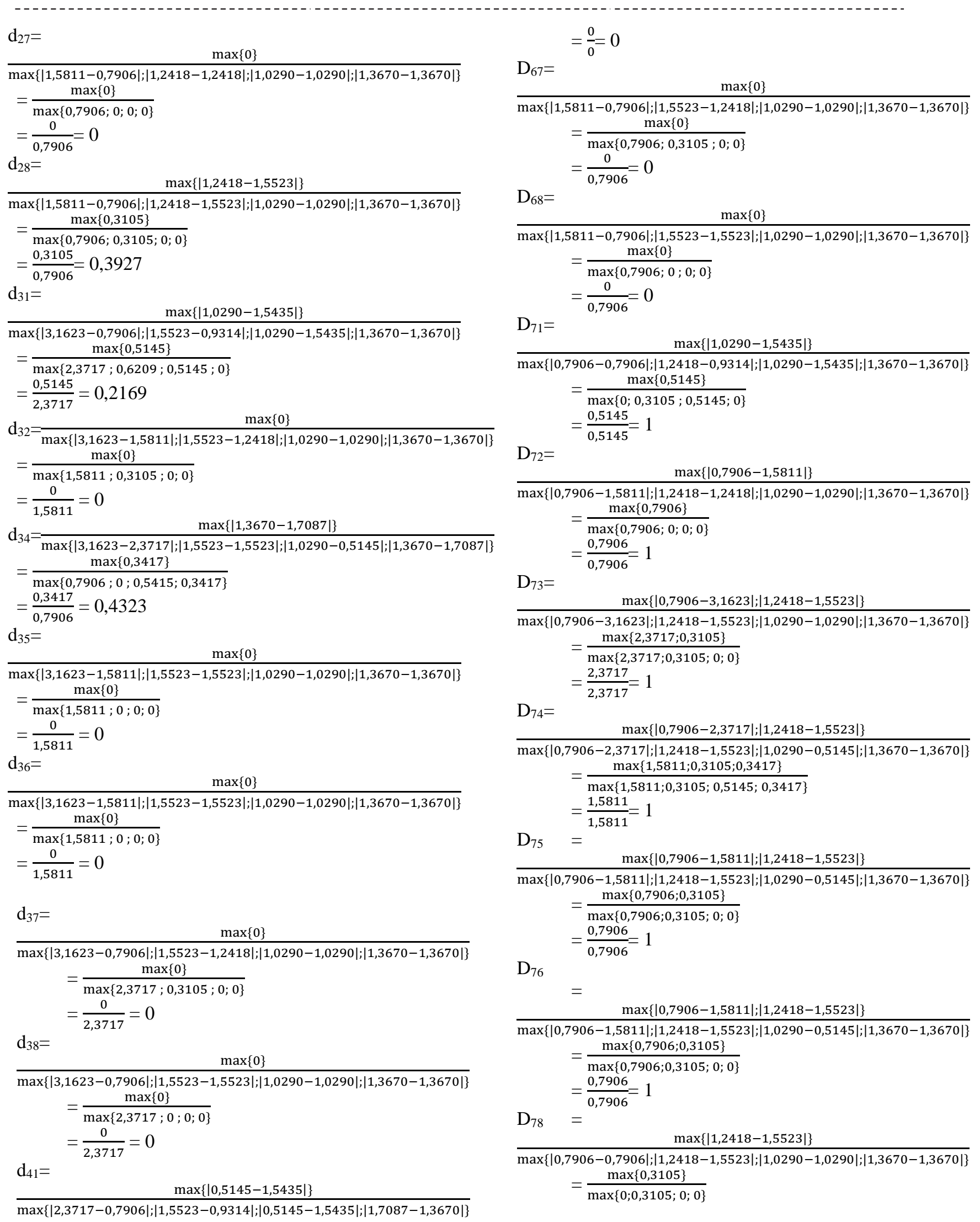




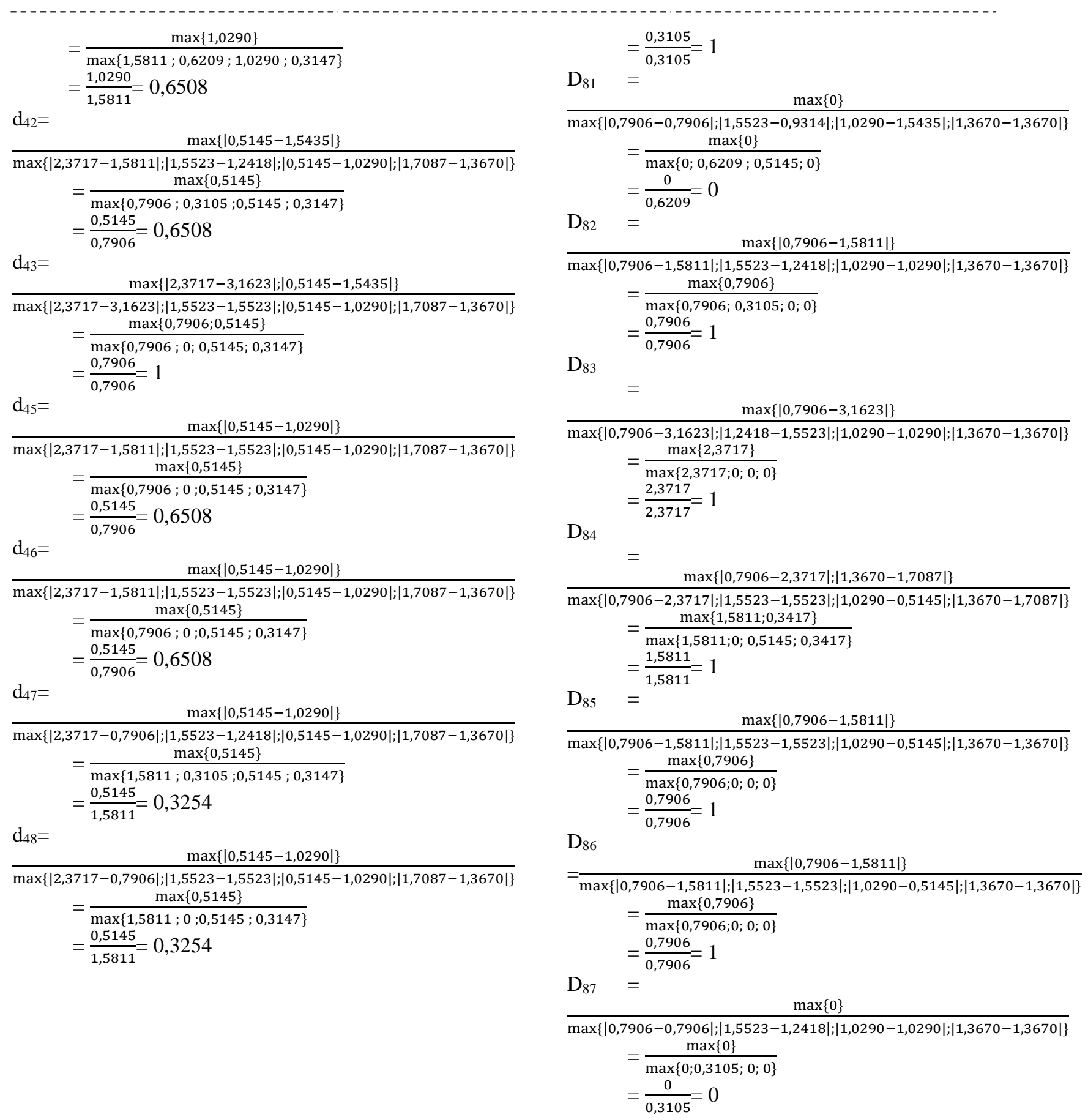

So the discordance matrix is: 
Journal of Computer Networks, Architecture and High Performance Computing e-ISSN 2655-9102, Volume 1, No. 2, Juli 2019, pp 45 -59

$\mathrm{D}=\left[\begin{array}{cccccccc}- & 1 & 1 & 1 & 1 & 1 & 0,6034 & 1 \\ 0,6508 & - & 1 & 1 & 1 & 1 & 0 & 0,3927 \\ 0,2169 & 0 & - & 0,4323 & 0 & 0 & 0 & 0 \\ 0,6508 & 0,6508 & 1 & - & 0,6508 & 0,6508 & 0 \times, 3254 & 0,3254 \\ 0,6508 & 0 & 1 & 0 & - & 0 & 0 & 0 \\ 0,6508 & 0 & 1 & 1 & 0 & - & 0 & 0 \\ 1 & 1 & 1 & 1 & 1 & 1 & - & 1 \\ 0,8286 & 1 & 1 & 1 & 1 & 1 & 0 & -\end{array}\right]$

6. Determine the Dominant Concordance Matrix and Dominant Discordan

a. Concordance

$$
\underline{\mathrm{C}}=\frac{651}{56}=11,6250
$$

Sehingga matriks dominan concordancenya adalah :

$$
\mathrm{F}=\left[\begin{array}{cccccccc}
- & 0 & 0 & 0 & 0 & 0 & 1 & 1 \\
1 & - & 0 & 0 & 1 & 1 & 1 & 1 \\
1 & 1 & - & 1 & 1 & 1 & 1 & 1 \\
1 & 1 & 0 & - & 1 & 1 & 1 & 1 \\
1 & 1 & 0 & 1 & - & 1 & 1 & 1 \\
1 & 1 & 0 & 0 & 1 & - & 1 & 1 \\
1 & 0 & 0 & 0 & 0 & 0 & - & 1 \\
1 & 0 & 0 & 0 & 0 & 0 & 1 & -
\end{array}\right]
$$

b. Discordance

$$
\underline{\mathrm{d}}=\frac{34}{56}=0,6077
$$

Sehingga matriks dominan discordancenya adalah :

$$
\mathrm{G}=\left[\begin{array}{cccccccc}
- & 1 & 1 & 1 & 1 & 1 & 0 & 1 \\
1 & - & 1 & 1 & 1 & 1 & 0 & 0 \\
0 & 0 & - & 0 & 0 & 0 & 0 & 0 \\
1 & 1 & 1 & - & 1 & 1 & 0 & 0 \\
1 & 0 & 1 & 1 & - & 0 & 0 & 0 \\
1 & 0 & 1 & 1 & 0 & - & 0 & 0 \\
1 & 1 & 1 & 1 & 1 & 1 & - & 1 \\
1 & 1 & 1 & 1 & 1 & 1 & 0 & -
\end{array}\right]
$$

7. Determine Aggregate Dominance Matrix

$$
\mathrm{F}=\left[\begin{array}{cccccccc}
- & 0 & 0 & 0 & 0 & 0 & 1 & 1 \\
1 & - & 0 & 0 & 1 & 1 & 1 & 1 \\
1 & 1 & - & 1 & 1 & 1 & 1 & 1 \\
1 & 1 & 0 & - & 1 & 1 & 1 & 1 \\
1 & 1 & 0 & 1 & - & 1 & 1 & 1 \\
1 & 1 & 0 & 0 & 1 & - & 1 & 1 \\
1 & 0 & 0 & 0 & 0 & 0 & - & 1 \\
1 & 0 & 0 & 0 & 0 & 0 & 1 & -
\end{array}\right] \times G=\left[\begin{array}{cccccccc}
- & 1 & 1 & 1 & 1 & 1 & 0 & 1 \\
1 & - & 1 & 1 & 1 & 1 & 0 & 0 \\
0 & 0 & - & 0 & 0 & 0 & 0 & 0 \\
1 & 1 & 1 & - & 1 & 1 & 0 & 0 \\
1 & 0 & 1 & 1 & - & 0 & 0 & 0 \\
1 & 0 & 1 & 1 & 0 & - & 0 & 0 \\
1 & 1 & 1 & 1 & 1 & 1 & - & 1 \\
1 & 1 & 1 & 1 & 1 & 1 & 0 & -
\end{array}\right]
$$

So that aggregate matrix dominancenya :

$$
\mathrm{E}=\left[\begin{array}{cccccccc}
- & 0 & 0 & 0 & 0 & 0 & 0 & 1 \\
1 & - & 0 & 0 & 1 & 1 & 0 & 0 \\
0 & 0 & - & 0 & 0 & 0 & 0 & 0 \\
1 & 1 & 0 & - & 1 & 1 & 0 & 0 \\
1 & 0 & 0 & 0 & - & 0 & 0 & 0 \\
1 & 0 & 0 & 0 & 0 & - & 0 & 0 \\
1 & 0 & 0 & 0 & 0 & 0 & - & 1 \\
1 & 0 & 0 & 0 & 0 & 0 & 0 & -
\end{array}\right]
$$

8. Elimination of Less Favorable Alternatives

Based matiks E, found that A4 (Ayla) has a value of 1 at most as many as 4 so that Ayla is the best alternative to be chosen.

2. System Results

a. Home Page

After the user has successfully logged in, the Home menu will appear first. This interface contains several navigation buttons. 


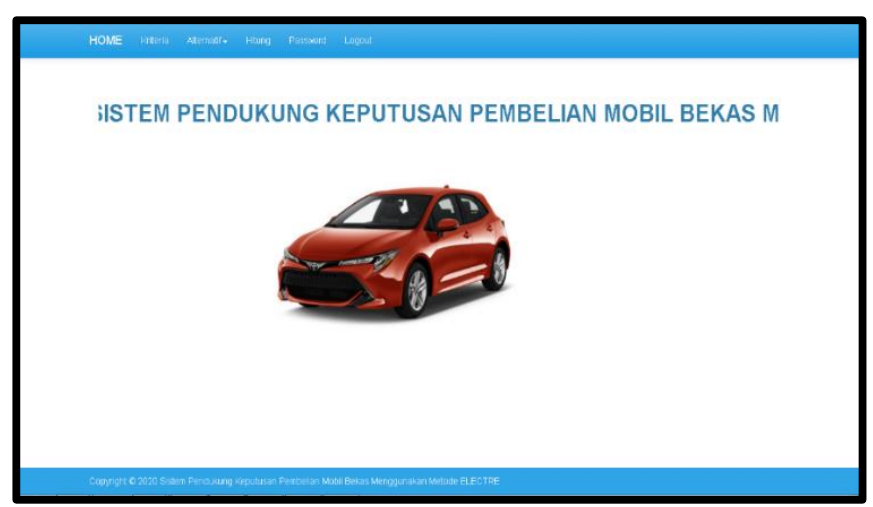

b. Criteria Page

Figure 1 Home page

When the user selects the criteria navigation button, a list of criteria will appear including the criteria code column, the criteria list, weights along with the print button to print the criteria report, the Edit button to

change the criteria data if needed, and the clear button to delete the criteria data from the storage media. Refresh button to refresh criteria page.

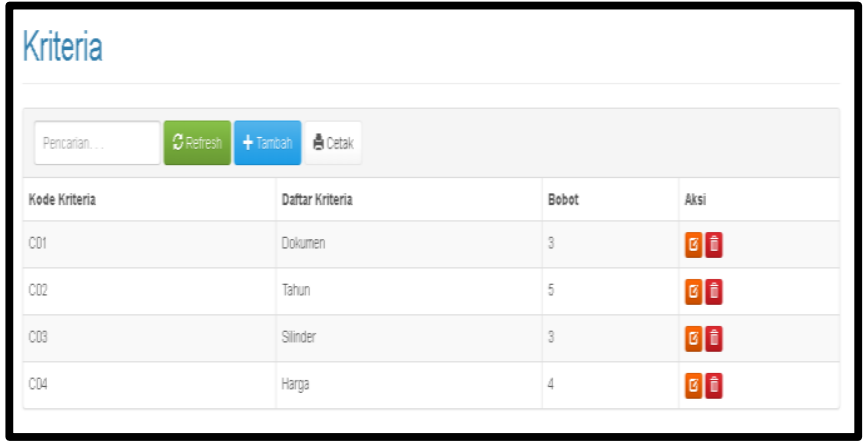

Figure 2 Criteria page

On the criteria page there is a navigation button "Add" to add new criteria. The criteria code will be filled in automatically, enter the criteria name and the weight then select save.

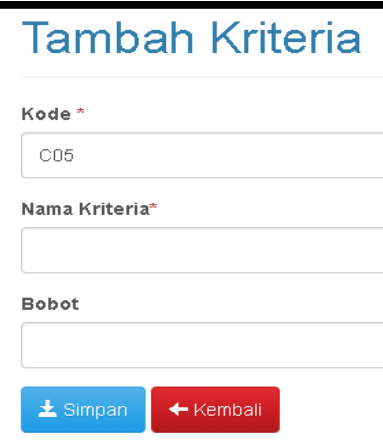

\section{c. Alternative Menu}

Figure 3 Add Criteria page

The Alternative Menu has two sub menus namely Alternative List and Alternative Value. The Alternative List page includes a code field, an alternative list, a print button to print alternative reports, an Edit button to change alternative data if needed, and a clear button to remove alternative data from storage. A refresh button to refresh the alternative list page. 


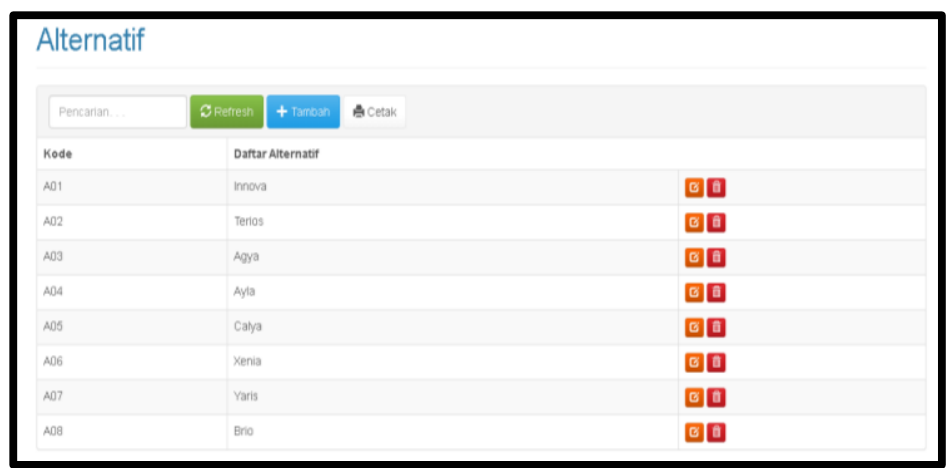

Figure 4 Alternative List Page

\section{d. Calculate Page}

The calculation page contains all calculation results using the ELECTRE method starting from the decision matrix, $\mathrm{R}$ matrix, $\mathrm{V}$ matrix, concordance, discordance, concordance matrix, discordance matrix, dominant concordance matrix, discordance dominant matrix, and aggregate dominance matrix E. Print button to print the result report ranking.

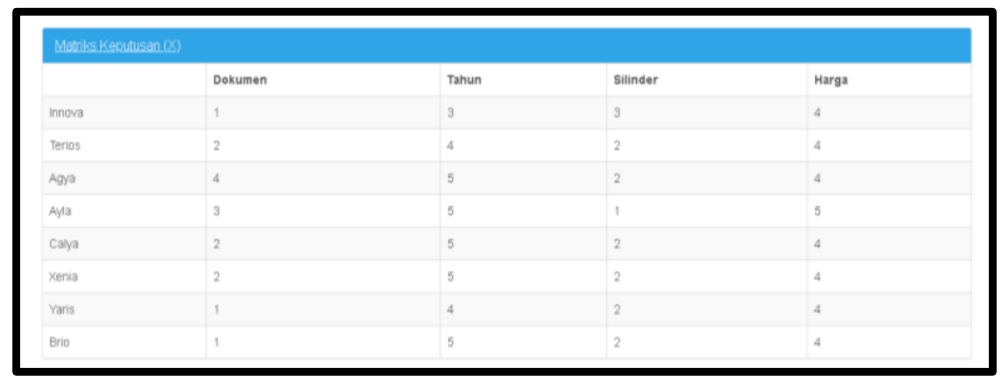

Figure 5 Count Page (Decision Matrix)

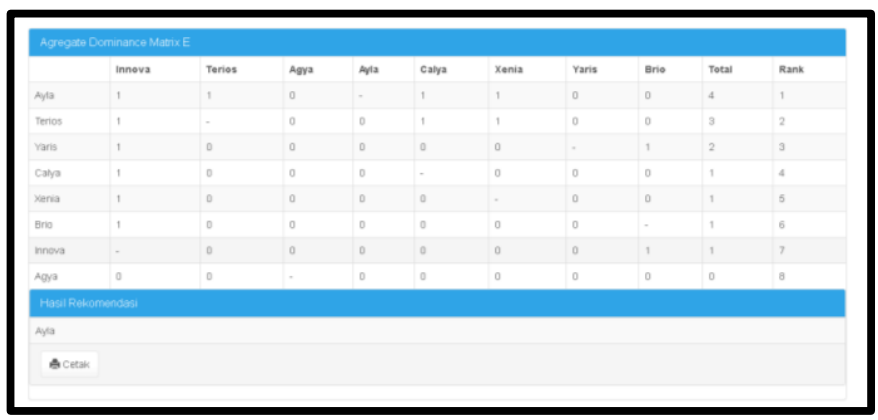

Figure 6 Count Pages (Aggregate Dominance Matrix E)

\section{Discussion}

Based on the E matrix, it is found that A4 (Ayla) has a value of 1 at most, which is 4 so that Ayla is the best alternative to choose. SPK for Used Car Purchases that are designed to operate properly and correctly and accurately because the output from manual analysis and analysis output with the SPK for Used Car Purchases is entirely appropriate.

\section{Conclusion}

Based on the results of research that has been conducted by researchers, it can be concluded that the Decision Support System which is applied using the ELECTRE method can facilitate the decision making of used car purchases. The process of buying used cars using the ELECTRE method, starts from determining the decision matrix, normalizing the decision matrix, determining the weighting of the normalized decision matrix, determining the set of concordance and discordance matrices, calculating the concordance and discordance matrices, determining the dominant concordance matrix and dominant discordance., determine the aggregate dominance matrix and then eliminate the 
less favorable alternatives. For further system development, it is hoped that additional criteria can be added according to the needs of system users so as to improve system performance. It is hoped that in the future this method can be further developed by using more and more extensive data processing for used cars, so that this SPK can really be used as a support for decision making for used car purchases. To get a better comparison of results, it is hoped that this used car purchasing decision support system will be tested and compared using other SPK methods.

\section{REFERENCES}

Beni, dkk, 2019, Penerapan Metode ELECTRE Sebagai Sistem Pendukung Keputusan Dalam Penerimaan Beasiswa, Buletin Utama Teknik Vol.14 No.3

Cahya, dkk, 2017, Sistem Pendukung Keputusan Dengan Menerapkan Metode ELECTRE Dalam Menentukan Prioritas Calon Debitur, Jurnal Antivirus Vol.11 No.2

Diana dan Yuliani, 2014, Sistem Pendukung Keputusan Penerimaan Anggota Himpunan Mahasiswa Jurusan Teknik Informatika Dengan Menggunakan Metode Electre (Studi Kasus : Sekolah Tinggi Teknologi Adisutjipto Yogyakarta), Vol.3 No.2

Dinil, dkk, 2019, Implementasi Metode ELECTRE Untuk Menentukan Kelayakan Pemberian Kredit Sepeda Motor Pada Perusahaan Leasing, KOMIK (Konferensi Nasionla Teknologi Informasi dan Komputer) Vol.3 No.1

Dony dan Yuli, 2019, Rancang Bangun Aplikasi Sistem Pendukung Keputusan Seleksi Pemilihan Destinasi Pariwisata Sumatera Barat Menggunakan Metode ELECTRE, Jurnal Teknologi Informasi dan Pendidikan Vol.12 No.1

Eko, dkk, 2014, Sistem Pendukung Keputusan Pemilihan Produk Unggulan Daerah Menggunakan Metode Enthropy dan Electre (Studi Kasus : Dinas Koperasi, Industri dan Perdagangan Kabupaten Lamongan), Vol.7 No.1

Elmi dan Novi, 2017, Sistem Pendukung Keputusan Penentuan Alternatif Produk Terbaik dengan Metode Electre, Indonesian Journal of Computer Science Vol.6 No.1

Fahmi, dkk, 2015, Implementasi Metode Electre Pada Sistem Pendukung Keputusan SNMPTN Jalur Undangan, Kumpulan Jurnal Ilmu Komputer (KLIK) Vol.2 No.2

Marlinda Linda, 2016, Sistem Pendukung Keputusan Pemilihan Tempat Wisata Yogyakarta Menggunakan Metode Elimination Et Choix Traduisant La Realita (ELECTRE), Seminar Nasional Sains dan Teknologi

Mesran, dkk, 2018, Implementasi Metode Electre Dalam Penentuan Karyawan Berprestasi (Studi Kasus : PT. Megarimas Sentosa), Jurnal Riset Sistem Informasi dan Teknik Informatika (JURASIK) Vol.3

Saefudin dan Irwan, 2018, Sistem Pendukung Keputusan Menentukan Lokasi Event PT. Mitra Panglima Sejahtera (MPS) Honda Pandeglang Menggunakan Metode Electre, Jurnal Sistem Informasi Vol.5 No.1 